Editorial

\title{
Artroplastia de Rodilla con asistencia Robótica y Navegación
}

\section{Total Knee Arthroplasty with Navegation and Robotic Assistance}

\author{
David Figueroa P. ${ }^{1}$ \\ ${ }^{1}$ Editor de la Revista RCHOT; Clínica Alemana de Santiago SA, \\ Vitacura, Región Metropolitana, Chile
}

Rev Chil Ortop Traumatol 2019;60:1-2.

La artroplastia total de rodilla (ATR), es una opción quirúrgica para aliviar el dolor y mejorar la función en pacientes con artrosis de rodilla. El objetivo de la cirugía es lograr una prótesis bien alineada con buen balance ligamentoso, minimizando así el desgaste y mejorando la supervivencia del implante.

En general, entre el $80 \%$ y el $89 \%$ de los pacientes, están satisfechos con sus resultados después de la ATR, con una buena supervivencia de implantes entre 10 a 15 años; sin embargo, todavía hay un grupo de pacientes que no están satisfechos.

En ese sentido, pueden participar muchos factores, entre otros, se ha atribuido a una alineación inadecuada de los componentes, inestabilidad persistente, inadecuada función y otros.

En los últimos años, se introdujo la navegación por computadora y la robótica para controlar las variables quirúrgicas a fin de obtener una mayor consistencia en la colocación del implante y alineación de los componentes postoperatorios optimizando los resultados clínicos. Esa tecnología también está hoy día presente en nuestro país y en Latino América.

La mayoría de los estudios clínicos disponibles han demostrado que el uso de la navegación está asociado con menos valores atípicos radiográficos después de la ATR, disminuyendo los llamados outliers. Varios estudios han comparado los resultados radiológicos de la ATR navegada con los resultados de la ATR utilizando la instrumentación estándar, y el seguimiento ha demostrado que la ATR asistida por navegación puede mejorar la alineación, especialmente en pacientes con deformidad severa. No obstante, muchos de esos estudios, están limitados por el diseño o por pequeñas cohortes. Mientras que algunos argumentan que el uso de la cirugía asistida por computadora depende de la experiencia del usuario, la cirugía asistida por computadora puede ayudar a los cirujanos con menos experiencia a lograr de manera confiable buenos resultados a medio plazo con una tasa menor de complicaciones.

Si bien la navegación quirúrgica ayuda a mejorar la planificación de los implantes, las herramientas robóticas han surgido como una herramienta para ayudar a refinar la ejecución quirúrgica. De esa manera, el control robótico de los gestos quirúrgicos puede mejorar aún más la precisión en la colocación del implante y/o habilitar nuevas características de diseño del implante. En la actualidad, las técnicas robóticas se utilizan cada vez más en artroplastia de rodilla uni compartimental y aparece en los últimos años la cirugía robótica asistida para ATR. Se ha demostrado que las guías de corte automáticas impulsadas por robot reducen el tiempo y mejoran la precisión en comparación con las guías de navegación en los procedimientos de corte femorales y tibial en modelos de cadáver. Sin embargo, los procedimientos de ATR con asistencia robótica, y las implicaciones clínicas de su propuesta todavía no están del todo aclarados.

La navegación por computadora y las herramientas robóticas en ATR mantienen la promesa de un mejor control de las variables quirúrgicas que influyen en el resultado clínico. Esas variables incluyen la colocación del implante, la alineación de la extremidad inferior, el equilibrio de los tejidos blandos y, potencialmente, el diseño y la fijación del implante.

Nuevos estudios con mejores diseños metodológicos, y el seguimiento del tiempo, nos dirán si la promesa de la cirugía robótica realmente se expresa en un cambio importante en el nivel de satisfacción de nuestros pacientes.

\section{Bibliografía}

1 Bautista M, Manrique J, Hozack WJ. Robotics in Total Knee Arthroplasty. J Knee Surg 2019; [Epub ahead of print]. Doi: $10.1055 / \mathrm{s}-0039-1681053$
Address for correspondence David Figueroa P., MD, PhD, Editor Revista RCHOT; Clínica Alemana de Santiago SA, Vitacura, Región Metropolitana, Chile (e-mail: dhfigueroa@gmail.com).
DOI https://doi.org/ 10.1055/s-0039-1685499. ISSN 0716-4548.
Copyright $\odot 2019$ by Thieme Revinter Publicações Ltda, Rio de Janeiro, Brazil

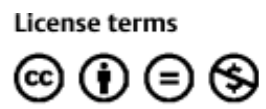


2 Sultan AA, Samuel LT, Khlopas A, et al. Robotic-Arm Assisted Total Knee Arthroplasty More Accurately Restored the Posterior Condylar Offset Ratio and the Insall-Salvati Index Compared to the Manual Technique; A Cohort-Matched Study. Surg Technol Int. 2019;34:sti34/1083. [Epub ahead of print]

3 Kayani B, Konan S, Tahmassebi J, Rowan FE, Haddad FS. An assessment of early functional rehabilitation and hospital discharge in conventional versus robotic-arm assisted unicompartmental knee arthroplasty: a prospective cohort study. Bone Joint J 2019;101-B(01):24-33

4 Cavinatto L, Bronson MJ, Chen DD, Moucha CS. Robotic-assisted versus standard unicompartmental knee arthroplasty-evaluation of manuscript conflict of interests, funding, scientific quality and bibliometrics. Int Orthop 2018; [Epub ahead of print]. Doi: 10.1007/s00264-018-4175-5

5 Kayani B, Konan S, Huq SS, Tahmassebi J, Haddad FS. Robotic-arm assisted total knee arthroplasty has a learning curve of seven cases for integration into the surgical workflow but no learning curve effect for accuracy of implant positioning. Knee Surg Sports Traumatol Arthrosc 2018; [Epub ahead of print]. Doi: 10.1007/ s00167-018-5138-5

6 Kayani B, Konan S, Tahmassebi J, Pietrzak JRT, Haddad FS. Roboticarm assisted total knee arthroplasty is associated with improved early functional recovery and reduced time to hospital discharge compared with conventional jig-based total knee arthroplasty: a prospective cohort study. Bone Joint J 2018;100-B(07):930-937

7 Deep K, Shankar S, Mahendra A. Computer assisted navigation in total knee and hip arthroplasty. SICOT J 2017;3:50. Doi: 10.1051/ sicotj/2017034

8 van der List JP, Chawla H, Joskowicz L, Pearle AD. Current state of computer navigation and robotics in unicompartmental and total knee arthroplasty: a systematic review with meta-analysis. Knee Surg Sports Traumatol Arthrosc 2016;24(11):3482-3495

9 Jacofsky DJ, Allen M. Robotics in Arthroplasty: A Comprehensive Review. J Arthroplasty 2016;31(10):2353-2363

10 Banerjee S, Cherian JJ, Elmallah RK, Jauregui JJ, Pierce TP, Mont MA. Robotic-assisted knee arthroplasty. Expert Rev Med Devices 2015;12(06):727-735. Doi: 10.1586/17434440.2015.1086264

11 Roche M. Robotic-assisted unicompartmental knee arthroplasty: the MAKO experience. Clin Sports Med 2014;33(01):123-132. Doi: 10.1016/j.csm.2013.08.007

12 Netravali NA, Shen F, Park Y, Bargar WL. A perspective on robotic assistance for knee arthroplasty. Adv Orthop 2013;2013:970703. Doi: $10.1155 / 2013 / 970703$

13 Stiehl J. Computer navigation and robotics. Knee 2013;20(04): 225. Doi: 10.1016/j.knee.2013.05.004 